Journal of Mathematics and Statistics 2 (3): 432-438, 2006

ISSN 1549-3644

(C) 2006 Science Publications

\title{
Computing the Moments of Order Statistics from Independent Non - Identically Distributed Burr Type XII Random Variables
}

\author{
A.A. Jamjoom \\ Department of Mathematics, Girls College of Education, Jeddah, Saudi Arabia
}

\begin{abstract}
In this paper, we derive a recurrence relation for computing all single moments of all order statistics arising from independent but not identically distributed Burr type XII random variables.
\end{abstract}

Keywords: Independent non - identical Variates; recurrence relations; order statistics, Moments, Burr distribution

\section{INTRODUCTION}

Let $X_{1}, X_{2}, \ldots, X_{n}$ be independent random Variables having cumulative distribution functions i.e. $F_{1}(x), F_{2}(x), \ldots, F_{n}(x)$ and probability density function, respectively.

Let $X_{1: n} \leq X_{2: n} \leq \ldots \leq X_{n: n}$ denote the order Statistics obtained by arranging the $\mathrm{n} X_{i^{\prime}{ }^{\prime}}$ in increasing order of magnitude . Bapat and Beg ${ }^{[1]}$ have shown that the CDF of the rth order Statistics $X_{r: n}(l \leq r \leq n)$ is conveniently expressed in terms of permanents as follows

$F_{r: n}(x)=\sum_{i=r}^{n} \frac{1}{i !(n-r) !} \operatorname{Per}\left[F(x) \quad \begin{array}{c}1-F(x)],-\infty<x<\infty \\ i\end{array}\right)$

where $F(x)$ and $1-F(x)$ denote the column vectors $\left(F_{1}(x), F_{2}(x), \ldots, F_{n}(x)\right)^{\prime}$ and $\left(1-F_{1}(x), 1-F_{2}(x), \ldots, 1-F_{n}(x)\right)^{\prime}$ respectively. Moreover if $a_{1}, a_{2}, \ldots$ are column vectors, then

$$
\left.\begin{array}{cc}
a_{1} & a_{2}
\end{array}\right],
$$

taking $i_{1}$ copies of $a_{1}, i_{2}$ copies of $a_{2}$ and so on. Also, in (1), Per (A) denotes the permanent of a square matrix A ; which is defined similarly as the determinant of A except that all terms in the expansion have a positive sign, i.e.

$$
\operatorname{Per} A=\sum_{\sigma \in S_{n}} \prod_{i=1}^{n} a_{i \sigma(i)}
$$

where $S_{n}$ is the set of permutations of $1,2, \ldots, \mathrm{n}^{[2,3,4]}$.

In the last few years much attention had been paid to order statistics from independent nonidentically distributed variables i.ni. $\mathrm{d}^{[5,6,7,8]}$.

Derivation of recurrence relations for single moments of order statistics from

i.ni.d available samples found in the literature have taken two directions, the work initiated by Balakrishnan [5,6] and that of Barakat and Abdelkader [9] In Balakrishnan 's work ${ }^{[5,6]}$, a linear relation between the PDF and CDF of the distribution, if exists, is exploited and then one has to go through messy calculations using integration by parts to get the result. Application of this method was done on many distributions such as: exponential $^{[5]}$, right-truncated exponential ${ }^{[6]}$ doublytruncated exponential and logistic distribution ${ }^{[10,11]}$, power function distribution ${ }^{[7]}$, Pareto and doublytruncated Pareto distributions ${ }^{[12]}$. All of these results were obtained by exploiting a basic differential equation satisfied by the distribution under consideration. For example: the differential equation satisfied by the PDF and CDF of exponential distribution is

$f_{i}(x)=\frac{1}{\theta_{i}}\left\{1-F_{i}(x)\right\}, x \geq 0, \theta_{i}>0 \quad i=1,2, \ldots, n$,

for Pareto distributions it is

$F_{i}(x)=1-\frac{x}{v_{i}} f_{i}(x) \quad i=1,2, \ldots, n$

and for power function distributions it is

$$
\begin{aligned}
& x f_{i}(x)=v_{i} F_{i}(x), 0 \leq x \leq 1 \\
& v_{i}>0, \\
& i=1,2, \ldots, n
\end{aligned}
$$


However, most of these recurrence relations show that it is enough to evaluate the kth moment of a single order statistics in a sample of size $n$, if these moments in samples of size less than $\mathrm{n}$ are already available. The kth moments of the remaining $n-1$ order statistics can then be determined by repeated use of these recurrence relations.

Barakat and Abdelkader ${ }^{[9]}$ generalized their procedure initiated in $(2000)^{[13]}$ to any d.f. and expressed the kth moment of the rth order statistics $\mu_{r: n}^{(k)}(k=1,2, \ldots),(1 \leq r \leq n)$ of a sample of size $\mathrm{n}$ purely in terms of the kth moments of the maximum order statistics or of the minimum order statistics from samples of size up to $\mathrm{n}$ of all possible subsamples of the given samples. This in fact simplifies the recursive computation of the single moments of (i.nid) order statistics.

Application of Barakat and Abdelkader 's method $^{[9]}$ started in fact in (2000) ${ }^{[13]}$

when they first applied it to calculate single moments of non-identically distributed Weibull random and in the year (2004) to Erlang distribution by ${ }^{[14]}$.

The advantages of their procedure can be simply described as follows: first there is no conditions imposed on the CDF. and PDF. of the underlying distribution, i.e. whether they are related on not; secondly $\mu_{r: n}^{(k)}(1 \leq r \leq n)$ obtained by their method is purely expressed in terms of the kth moments of maximums and the minimums of all possible subsamples of the given sample.

In this paper we consider the case where the r.v.s $X_{i}, i=1,2, \ldots, n$ are independent and non identical having Burr type XII distribution with CDF.

$$
F_{i}(x)=1-\left(1+x^{2}\right)^{-m_{i}}, x \geq 0, \quad c, m \geq 1
$$

For $i=1,2, \ldots, n$, where $\mathrm{c}$ and $m_{i}$ are shape parameters $^{[15,16,17,18,19]}$. We consider Burr type XII distribution since it is widely used in approximation, and as failure rate model ${ }^{[20]}$ and also in predication ${ }^{[21,22]}$, and in many other fields ${ }^{[23,24,25,26]}$. It has the advantage of being used in approximating distributions of rather complicated PDF's (i.e. intractable distributions $)^{[27,28,29,30,31]}$. Burr distribution, also known as Lomax at $\mathrm{c}=1$ or compound Weibull or Weibull Gamma distribution $^{[32]}$. At $\mathrm{m}=1$, the Burr distribution reduces to loglogistic or Weibull- Exponential distribution Al-shboul and Khan ${ }^{[33,34,35]}$.
In the next section we derive the kth moment of the largest order statistics $\mu_{n: n}^{(k)}=E\left(X_{n: n}^{k}\right)$ and smallest order statistics $\mu_{1: n}^{(k)}=E\left(X_{1: n}^{k}\right)$. Moreover, a recurrence relation is introduced which will enable one to compute the kth moments of all order statistics $\left(\mu_{r: n}^{(k)}\right.$, for all $\left.r \leq n\right)$ in a simple manner by using only the kth moments of the maximum.

_Relations for single moments: We shall present some recurrence relations for the single moments of order statistics obtained from Burr type XII distributions.

Relation 2.1: For $n=1,2, \ldots$ and $k=1.2, \ldots$.

$\mu_{1: n}^{(k)}=\frac{k}{c} I_{n}$

$\mu_{n: n}^{(k)}=\frac{k}{c} \sum_{j=1}^{n}(-1)^{j+1} I_{j}$

where

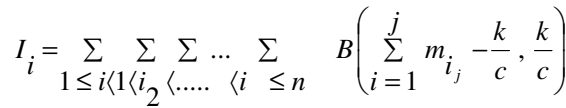

and $\mathrm{B}(\mathrm{c}, \mathrm{k})$ is the regular beta function define by

$$
B(c, k)=\frac{\Gamma(c) \Gamma(k)}{\Gamma(c+k)} .
$$

$$
\begin{aligned}
& \text { Proof } \\
& \text { Since } \\
& x_{i 0}=\inf \left\{x: F_{i}(x)\right. \\
& \text { definition of moments } \\
& \begin{aligned}
\mu^{(k)}=E\left(x^{k}\right) \\
=\int_{0}^{\infty} x^{k} f(x) d x \\
=\int_{0}^{\infty} x^{k} d F(x) \\
=-\int_{0}^{\infty} x^{k} d(1-F(x)),
\end{aligned}
\end{aligned}
$$

$x_{i 0}=\inf \left\{x: F_{i}(x) \succ 0\right\} \geq 0, i=1,2, \ldots, n$, then by definition of moments we have:

Integrating by part gives

$\mu^{(k)}=k \int_{0}^{\infty} x^{k-1}[1-F(x)] d x$ 
This equation was obtained by Galambos ${ }^{[36]}$. Then the kth moment of the smallest is

$\therefore \mu_{1: n}^{(k)}=k \int_{0}^{\infty} x^{k-1}\left(1-F_{1: n}(x)\right) d x$

where $F_{1: n}(x)$ is the CDF of the smallest order statistics from independent not identically distributed random variables defined by

$F_{1: n}(x)=1-\prod_{i=1}^{n}\left(1-F_{i}(x)\right)$

$\left(1-F_{1: n}(x)\right)=\prod_{i=1}^{n}\left(1-F_{i}(x)\right)$

substituting (10) in (8) we get

$\mu_{1: n}^{(k)}=k \int_{0}^{\infty} x^{k-1} \prod_{i=1}^{n}\left(1-F_{i}(x)\right) d x$

Now substituting (2) in (11) we get

$$
\begin{aligned}
\mu_{1: n}^{(k)} & \left.=k \int_{0}^{\infty} x^{k-1} \prod_{i=1}^{n}\left(1+x^{c}\right)\right)^{-m_{i}} d x \\
& \left.=k \int_{0}^{\infty} x^{k-1}\left(1+x^{c}\right)\right)^{-} \sum_{i=1}^{n} m_{i} d x
\end{aligned}
$$

upon using

$$
\int_{0}^{\infty} x^{k-1}\left(1+x^{c}\right)^{-\alpha} d x=\frac{1}{c} B\left(\alpha-\frac{k}{c}, \frac{k}{c}\right)
$$

where $B(c, k)$ is the regular beta function

$$
\therefore \mu_{1: n}^{(k)}=\frac{k}{c} B\left(\sum_{i=1}^{n} m_{i}-\frac{k}{c}, \frac{k}{c}\right)
$$

which can be written as

$$
\mu_{1: n}^{(k)}=\frac{k}{c} I_{n}
$$

where

$$
I_{n}=B\left(\sum_{i=1}^{n} m_{i}-\frac{k}{c}, \frac{k}{c}\right)
$$

which can also be written as

$$
I_{n}=\sum_{1 \leq i_{1}\left\langlei _ { 2 } \left\langle\ldots<i_{n} \leq n\right.\right.} \sum_{i=1} B\left(\sum_{i=1}^{n} m_{i_{n}}-\frac{k}{c}, \frac{k}{c}\right)
$$

where the symbol $\sum_{1 \leq i_{1} \prec i_{2} \prec} \sum_{\ldots .<i_{n}} \ldots \sum_{\leq n} l_{i_{n}}$ denote to the sum of the $l_{n}$ from all possible subsamples of size $\mathrm{n}$ ( which is one sample in this case ) of the given sample The proof of (4) follows:

$$
\mu_{n: n}^{(k)}=k \int_{0}^{\infty} x^{k-1}\left[1-F_{n: n}(x)\right] d x
$$

where $F_{n: n}(x)$ the CDF of the largest order statistics from independent not identically distributed random variable defined by

$$
F_{n: n}(x)=\prod_{i=1}^{n} F_{i}(x)
$$

and for Burr Type XII it is

$$
F_{n: n}(x)=\prod_{i=1}^{n}\left[1-\left(1+x^{c}\right)^{-m_{i}}\right]
$$

$$
\begin{aligned}
\therefore \mu_{n: n}^{(k)}=k \int_{0}^{\infty} x^{k-1}\left[1-\prod_{i=1}^{n}\left[1-\left(1+x^{c}\right)^{-m_{i}}\right]\right] d x & {\left[\begin{array}{l}
\left.\sum_{i=1}^{n}\left(1+x^{c}\right)^{-m_{i}}-\sum_{1 \leq i_{1}\left\langle i_{2} \leq n\right.} \sum_{0}\left(1+x^{c}\right)^{-\left(m_{i_{1}}+m_{i_{2}}\right)}\right] \\
=k \int_{0}^{\infty} x^{k-1}\left[\begin{array}{l}
+\sum_{1 \leq i_{1}\left\langlei _ { 2 } \left\langle i_{3} \leq n\right.\right.} \sum^{\sum \ldots \ldots \ldots .}\left(1+x^{c}\right)^{-\left(m_{i_{1}}+m_{i}+m_{i_{3}}\right)} \\
+\ldots \ldots
\end{array}\right] d x
\end{array}\right] }
\end{aligned}
$$

Using (13) we get 
$\mu_{n: n}^{(k)}=\frac{k}{c}\left[\begin{array}{l}\sum_{i=1}^{n} B\left(\sum_{i=1}^{n} m_{i}-\frac{k}{c}, \frac{k}{c}\right) \\ -\sum_{1 \leq i_{1}\left\langle i_{2} \leq n\right.} B\left(m_{i_{1}}+m_{i_{2}}-\frac{k}{c}, \frac{k}{c}\right) \\ +\sum_{1 \leq i_{1}\left\langle i_{2}\left\langle i_{3} \leq n\right.\right.} B\left(m_{i_{1}}+m_{i_{2}}+m_{i_{3}}-\frac{k}{c}, \frac{k}{c}\right) \\ +\ldots \ldots . . \\ +(-1)^{n+1} B\left(\sum_{i=1}^{n} m_{i}-\frac{k}{c}, \frac{k}{c}\right)\end{array}\right]$

$\mu_{n: n}^{(k)}=\frac{k}{c} \sum_{j=1}^{n}(-1)^{j+1} I_{j}$

where

$\mathrm{I}_{j}=\sum_{1 \leq i_{1}\left\langle i_{2}\left\langle\ldots i_{j} \leq n\right.\right.} \sum_{i=1} \beta\left(\sum_{i=1}^{j} m_{i}-\frac{k}{c}, \frac{k}{c}\right)$

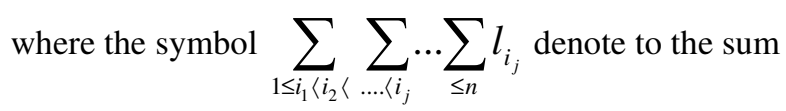

of the $l_{j}$ from all possible subsamples of size $\mathrm{j}$ of the

given sample (which are $\left(\begin{array}{l}n \\ j\end{array}\right)$ samples in this case).

Relation 2.2. For $r=1,2, \ldots ., n$ and $k=1,2, \ldots$.

$\mu_{r: n}^{(k)}=\mu_{r-1: n}^{(k)}+J_{r: n}^{(k)}$

where

$J_{r: n}^{(k)}=\sum_{j=1}^{r}(-1)^{j-1} \frac{k}{c} a_{j} \mathrm{I}_{n-r+j}$

where

$a_{j}=\frac{(n-r+j) !}{(n-r+1) !(j-1) !}$ and the sequence $\left\{\mathrm{I}_{j}\right\}_{j=1}^{j=r}$ is defined in relation

(3) .and $\mu_{o: n}^{(k)}=0$ for convention .

Proof: If we replace $r$ with $(r-1)$ in equation (1.1) we get

$F_{r-1: n}(x)=\sum_{i=r-1}^{n} \frac{1}{i !(n-i) !} \operatorname{Per}\left[\begin{array}{cc}F(x) & 1-F(x) \\ i & n-i\end{array}\right]$

expanding the summation on the first term, then

$F_{r-1: n}(x)=F_{r: n}(x)+\frac{1}{(r-1) !(n-r+1) !} \operatorname{Per}\left[\begin{array}{rr}F(x) & 1-F(x) \\ r-1 & n-r+1\end{array}\right]$

which is equivalent to

$F_{r-1: n}(x)=F_{r: n}(x)+\sum_{p} \prod_{j=1}^{r-1} F_{i j}(x) \prod_{j=r}^{n}\left(1-F_{i}{ }_{n-j+1}(x)\right)$

where the summation $\mathrm{P}$ extends over all permutation $\left(i_{1}, i_{2}, \ldots ., i_{n}\right)$ of $(1,2, \ldots ., n)$ for which $1 \leq i_{1}<i_{2}<\ldots<i_{n-1} \leq n$ and $1 \leq i_{r}\left\langle i_{r+1}\left\langle\ldots .<i_{r-1} \leq n\right.\right.$. Now since $x_{i 0}=\inf \left\{x: F_{i}(x)>0\right\} \geq 0$, for all $i$, then

$\mu_{r: n}^{(k)}=E\left(X_{r: n}^{(k)}\right)=k \int_{0}^{\infty} x^{k-1}\left(1-F_{r: n}(x)\right) d x$

substituting (17) in (18)

$$
\begin{aligned}
& \mu_{r: n}^{(k)}=k \int_{0}^{\infty} x^{k-1}\left[\begin{array}{l}
1-F_{r-1: n}(x)+\sum_{p j=1}^{r-1} F_{i j}(x) . \\
\prod_{j=r}^{n}\left(1-F_{i_{n-j+1}}(x)\right) d x
\end{array}\right] \\
& \quad k \quad \int_{0}^{\infty} x^{k-1}\left(1-F_{r-1: n}(x)\right) d x+ \\
& \quad+k \sum_{p}^{\infty} \int_{0}^{k-1} \prod_{j=1}^{r-1} F_{i j}(x) \prod_{j=r}^{n}\left(1-F_{i_{n-j+1}}(x)\right) d x \\
& \therefore \mu_{r: n}^{(k)}=\mu_{r-1: n}^{(k)}+J_{r: n}^{(k)}
\end{aligned}
$$

where

$$
J_{r: n}^{(k)}=k \sum_{p} \int_{0}^{\infty} x^{k-1} \prod_{j=1}^{r-1} F_{i j}(x) \prod_{j=r}^{n}\left(1-F_{i_{n-j+1}}(x)\right) d x
$$

Now consider 


$$
\begin{aligned}
& F_{i}(x)=1-\left(1+x^{c}\right)^{-m}, x \geq 0, \text { it follows } \\
& \prod_{j=1}^{r-1} F_{i_{j}}(x) \prod_{j=r}^{n}\left(1-F_{i_{n-j+1}}(x)\right)=\prod_{j=1}^{r-1}\left[1-\left(1+x^{c}\right)^{-m_{i}}\right] \prod_{j=r}^{n}\left(1+x^{c}\right)^{-m_{i_{j}}} \\
& =\prod_{j=1}^{r-1}\left[1-\left(1+x^{c}\right)^{-m_{i}}\right]\left(1+x^{c}\right)^{-} \sum_{j=r}^{n} m_{i}
\end{aligned}
$$

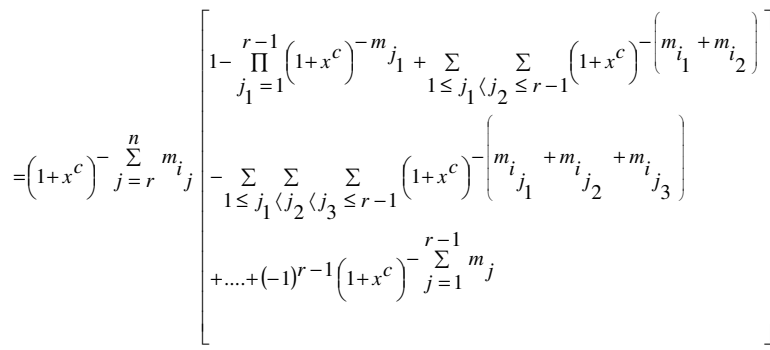

$$
\begin{aligned}
& \prod_{j=1}^{r-1} F_{i_{j}}(x) \prod_{j=r}^{n}\left(1-F_{i_{i}}(x)\right)=\left(1+x^{c}\right)^{-}-\sum_{j=r}^{n} m_{i_{j}}-\sum_{j_{1}=1}^{r-1}\left(1+x^{c}\right)-\left(m_{i_{j_{1}}}+\sum_{j=r}^{n} m_{i_{j}}\right)
\end{aligned}
$$

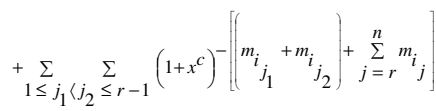

$$
\begin{aligned}
& \left.\left.-\sum_{1 \leq j_{1} \backslash j_{2}\left\langle j_{3} \leq r-1\right.}\left(1+x^{c}\right)^{-\left[m_{i_{j_{1}}}\right.}+m_{i_{j_{2}}}+m_{i_{j_{3}}}\right)+\sum_{j=r}^{n} m_{i_{j}}\right]
\end{aligned}
$$

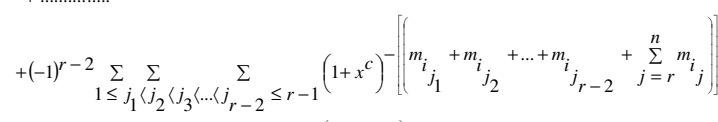

$$
\begin{aligned}
& +(-1)^{r-1}\left(1+x^{c}\right)-\left(\sum_{j=1}^{n-1} m_{i}\right),
\end{aligned}
$$

Substituting (20) in (19) and after simple calculation we get

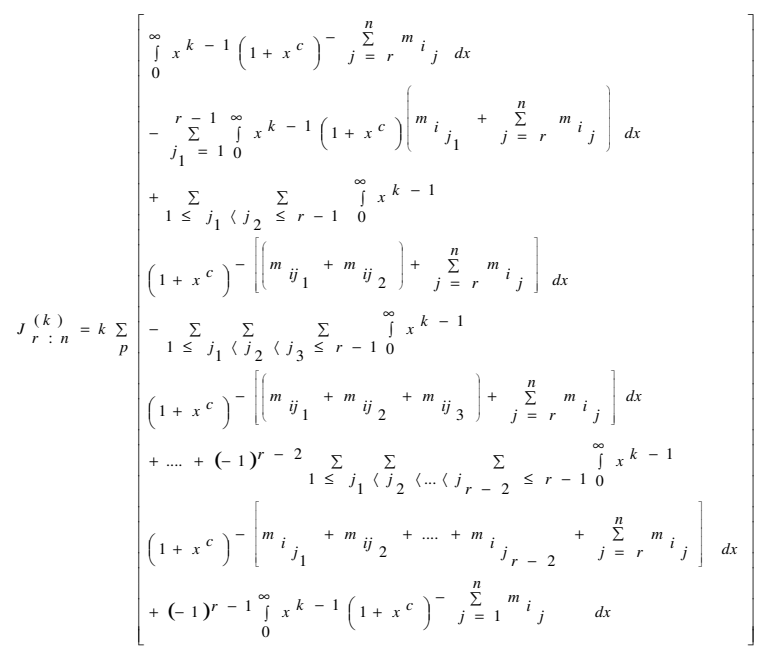

Upon using the integration (13) we get

$$
\begin{aligned}
& J_{r: n}^{(k)}=\frac{k}{c} \sum_{p}\left[B \sum_{j=r}^{n} m_{i j}-\frac{k}{c}, \frac{k}{c}\right] \\
& -\sum_{j}^{r-1} B\left[\left(m_{i i_{1}}+\sum_{j=r}^{n} m_{i j}\right)-\frac{k}{c}, \frac{k}{c}\right] \\
& +\sum_{1 \leq j_{1}\left\langle j_{2} \leq r-1\right.} \sum^{\sum} B\left[\left(m_{i_{j_{1}}}+m_{i_{j_{2}}}\right)+\sum_{j=r}^{n} m_{i j}-\frac{k}{c}, \frac{k}{c}\right] \\
& -\underset{1 \leq j_{1}<j_{2}\left\langle j_{3} \leq r-1\right.}{\sum} B\left[\left(m_{i_{j_{1}}}+m_{i_{j_{2}}}+m_{i_{j_{3}}}\right)+\sum_{j=r}^{n} m_{i j}-\frac{k}{c}, \frac{k}{c}\right] \\
& +\ldots . .+(-1)^{r-2} \sum_{1 \leq j_{1}\left\langlej _ { 2 } \left\langlej _ { 3 } \left\langle\ldots j_{r-2} \leq r-1\right.\right.\right.} \sum^{2} \\
& B\left[\left(m_{i_{j_{1}}}+m_{i_{j_{2}}}+\cdots+m_{i_{j_{r-2}}}\right)+\sum_{j=r}^{n} m_{i j}-\frac{k}{c}, \frac{k}{c}\right] \\
& +(-1)^{r-1} \beta\left[\sum_{j=1}^{n} m_{i j}-\frac{k}{c}, \frac{k}{c}\right]
\end{aligned}
$$

By using the fact that $\sum_{p}(1)=\left(\begin{array}{c}n \\ r-1\end{array}\right)$ and

$\sum_{1 \leq j_{1}\left\langle j_{2}\left\langle j_{3}\left\langle\ldots \leq j_{m} \leq n\right.\right.\right.} \sum_{m}\left(\begin{array}{l}n \\ m\end{array}\right)$ for all $n \geq m$

we get

$J_{r: n}^{(k)}=\sum_{j=1}^{n}(-1)^{j-1} \frac{k}{c} a_{j} \mathrm{I}_{n-r+j}$

where the sequence $\left\{\mathrm{I}_{j}\right\}_{j=n-r+1}^{j=n}$ is defined in

(5) and $a_{j}=\frac{(n-r+j) !}{(n-r+1) !(j-1) !}$

since

$\left(\begin{array}{c}n \\ r-1\end{array}\right)\left(\begin{array}{c}r-1 \\ j-1\end{array}\right)=a_{j}\left(\begin{array}{l}n \\ r-1\end{array}\right)$

which completes the proof.

\section{CONCLUSION}

By recursively applying equation (5) starting with the maximum $\mu_{n: n}^{(k)}$ in (4) one can deduct all moments of all order statistics $\mu_{r: n}^{(k)}, r \leq n$ from Burr type XII distributions. One only needs to compute the sequence $\left\{I_{j}\right\}_{j=1}^{n}$ which is given by (5). This sequence is very simple to evaluate . For example if $n=3$, we get 
$\mu_{3: 3}^{(k)}=\frac{k}{c}\left(I_{1}-I_{2}+I_{3}\right)$

where

$I_{1}=B\left(m_{1}-\frac{k}{c}, \frac{k}{c}\right)+B\left(m_{2}-\frac{k}{c}, \frac{k}{c}\right)+B\left(m_{3}-\frac{k}{c}, \frac{k}{c}\right)$

$I_{2}=B\left(m_{1}+m_{2}-\frac{k}{c}, \frac{k}{c}\right)+B\left(m_{1}+m_{3}-\frac{k}{c}, \frac{k}{c}\right)+B\left(m_{2}+m_{3}-\frac{k}{c}, \frac{k}{c}\right)$

$I_{3}=B\left(m_{1}+m_{2}+m_{3}-\frac{k}{c}, \frac{k}{c}\right)$

$\mu_{1: 3}^{(k)}=\frac{k}{c} I_{3}$

$\mu_{2: 3}^{(k)}=\frac{k}{c}\left[I_{2}-3 I_{3}\right]$

These results can be put in the following table

The moments $\mu_{r: n}^{(k)}, r \leq n$ of order statistics arising from non-identically Burr type XII random variables with $n=3$

$\begin{array}{lccc}\mu_{3: 3}^{*(K)} & I_{1} & -I_{2} & +I_{3} \\ \mu_{2: 3}^{*(K)} & & I_{2} & -2 I_{3} \\ \mu_{1: 3}^{*(K)} & & & I_{3}\end{array}$

$\mu_{r: n}^{*(k)}=\frac{c}{k} \mu_{r: n}^{(k)}$. Generalization of this table is mentioned in ${ }^{[13]}$.

\section{REFERENCES}

1. Bapat, R. and M. Beg, 1989. Order Statistics from non-identically distributed variables and permanents. Sankh ${ }^{\sim}$ a, A, 51 : 79-93.

2. Minc, H., 1978. Permanents, Encyclopedia of Mathematics and its applications 6, AddisonWesley, Reading, MA

3. Minc, H., 1983. Theory of permanents (1978 1981). Linear and multilinear Algebra, 12: 227-263.

4. Minc, H., 1987. Theory of permanents (1982 1985). Linear and multilinear Algebra, 12: 227-263.

5. Balakrishnan, N., 1994 a. Order statistics from non-identically exponentically random variables and some applications. Comput. Statist. Data- Anal. 18, No. 2: 203-225.

6. Balakrishnan, N., 1994b. On order statistics from non-identical right-truncated exponential random variables and some applications. Commun. Statist. - Theory Meth. 23 : 33733393

7. Balakrishnan, N. And K. Balasubramanian, 1995. Order ststistics from non-identically Power function random variables. Commun. Statist.-Theory Meth., 24(6) : 1443-1454.
8. Coa, G. and M. West, 1977. Computing distributions of order Statistics. Common. Statist. - Theory meth. 26, $3:$ 755-764.

9. Barakat, H. And Y. Abdelkader, 2004. Computing the Moments of order statistics from nonidentical random variables. Statistics Methods \& Applications.13: 15-26.

10. Childs, A. and N. Balakrishnan, 1995a. Generalized recurrence relations for moments of order statistics from non-identical doublytruncated random variables. Preprint.

11. Childs, A. and N. Balakrishnan, 1995b. Relations for single moments of order statistics from non-identical logistic random variables and assessment of the effect of multiple outliers on the bias of linear estimators of location and scale. Preprint.

12. Childs, A. and N. Balarishnan, 1998. Generalized recurrence relations for moments of order statistics from non-identical Pareto and truncated Pareto random variables with applications to robustness. In: Balakrishnan N, Rao RC (eds) Handbook of statistics, 16, North-Holland, Amsterdam: 403-438.

13. Barakat, H. and Y. Abdelkader, 2000. Computing the moments of order statistics from nonidentically distributed Weibull variables. J. Comp. Appl. Math. 117, $1: 85-90$

14. Abdelkader, Y., 2005. Computing the moments of order statistics from nonidentically distributed Erlang variables. Statistical Papers, .45, 4 :563-570.

15. Burr, I .W., 1942. Cumulative frequency functions. Annals of mathematical statistics, 13: 215-232.

16. Burr, I.W., 1967. A Useful Approximation to the Normal Distribution Function, with Application to

Simmulation. Technometrics, .9, $4:$ 647-651.

17. Burr, I.W., 1968. On a General system of Distributions. III the Sample Range. Journal American Statist. Assn ., 62 : 637-643.

18. Burr, I .W., and P. Cislak, 1968. On general system of distribution I. Its curve shape characteristics II .The Sample Median. Jour. Amer. Statist. Assn. 62: $627-635$.

19. Rodriguez, N.R., 1977. A Guide to the Burr type XII distribution. Biometrika, 54, 1: 129 134.

20. Gupta, P.L, R.C. Gupta and S. J. Lvin., 1996. Analysis of failure time data by Burr distribution". Common. Statist Theory Meth., 25 ,9: $2013-2024$.

21. Ashour, S .K., and M. El - Wakeel, 1993. Bayesian prediction of the jth order statistics with Burr distribution and random sample size. Microelectron . Reliab., 33, 8 : 1179 1188. 
22. Nigm, A.M., and N.Y. Abd Al - Wahab, 1996. Bayesian prediction with a random sample size for the Burr life distribution. Commun Statist Theory Meth. 25 (6) : 1289 - 1303.

23. Papdopoulos, A.S., 1978. The Burr distribution as a failure model from a Bayesian approach". IEEE Trans. On Rel. 5 : 369 - 371

24. Evans, I.G., and A. Ragab, 1983. Bayesian inferences given a type -2 censored sample from a Burr distribution. Commun. Statist. th. Methods A, 12 (1): 1569 - 1580.

25. Pandey, $M$ and Borhan Uddin, 1991. Estimation of reliability in Multi - Component stress - strength model following a Burr distribution". Microelectron. Reliab., 31, $1: 21$ -25 .

26. Wingo, D.R., 1993b. Maximum likelihood methods for fitting the Burr type XII distribution to multiply (Progressively) censored life test data. Metrika, $40: 203-210$

27. Austin Jr., J.A., 1973. Control Chart Constants for largest and smallest in a sampling from a normal Distribution Using the Generalized Burr Distribution. Technometrics, 15, 4 :130933.

28. Tadikamalla, P.R., and J. Ramberg, 1975. An Approximate Method for Generating Gamma and Other Variates. J.Statist Comput. Simul., $3: 375-382$
29. Wheeler, D., 1975. An Approximation for Simulation of Gamma Distributions. Statist. Comput. Simul., 3 : 225- 232

30. Tadikamalla, P. R., 1977. An Approximation to the Moments and the Percentiles of Gamma Order Statistics . Sankya: The Indian Journal of Statistics, 39, Series B, pt. 4 : 372-381.

31. Tadikamalla, P.R., 1980. A Look at the Burr and Related Disatribution . Intl . Statistical Review, 48 : 337-344.

32. Lomax, K.S., 1954. Business Failures: AnotherExample of the Analysis of Failure Data. J. American Statist Assoc.: 847-852.

33. Al-Shboul, Q.M. and A. Khan, 1989 . Moments of order statistic from doubly truncated log-logistic distribution. Pakistan J. Statist ., 5(3) B : 209-307.

34. Khan, A.H. and I. Khan, 1987. Moments of order statistics from Burr distribution and its characterizations Metron, XL V-N, 1-2 : 21-29

35. Ragab, A. And J. Green, 1984. On Order Statistics From the Log-Logistic Distribution and Their Properties .Comm.in Statistics, A13, 21.

36. Galambos, J., 1987. The asympototic theory of extreme order statistics, $2^{\text {nd }}$ edn. Krieger, Malabar, Florida. 